\title{
Recent developments in the application of X-ray and neutron reflectivity to soft matter systems
}

\author{
Maximilian W.A. Skoda
}

\section{Published version information}

Citation: MW Skoda. "Recent developments in the application of X-ray and neutron reflectivity to soft matter systems." Current Opinion in Colloid and Interface Science, vol. 42 (2019): 41-54.

DOI: $\underline{10.1016 / \text { i.cocis.2019.03.003 }}$

C2019. This manuscript version is made available under the CC-BY-NC-ND 4.0 Licence.

This version is made available in accordance with publisher policies. Please cite only the published version using the reference above. This is the citation assigned by the publisher at the time of issuing the AAM. Please check the publisher's website for any updates. 


\title{
Recent developments in the application of X-ray and neutron reflectivity to soft matter systems
}

\author{
Maximilian W. A. Skoda ${ }^{a}$ \\ ${ }^{a}$ Rutherford-Appleton Laboratory, ISIS Facility, Didcot, OX11 OQX, United Kingdom
}

\begin{abstract}
Many interesting physical, chemical and biological phenomena occur at interfaces between nanometre scale layers of soft condensed matter. Theses often complex systems lend themselves to study by X-ray (XRR) and neutron reflectivity (NR). The application of these techniques to such systems is extremely wide spread and provides unique insights into their structure and dynamics. This review presents a snapshot of recent activity in this research area and identifies trends in the application of XRR and NR to novel, unusual or highly complex sample systems. Although the majority of research using these techniques is investigating variations on 'traditional' systems, supported by progress in instrumentation, advance sample environment and computational tools, NR and XRR have begun to produce singular insights in areas such as atmospheric science, real biological systems (cells and bacteria), oil-water interfaces or industrial problems (rheology, packaging or durability of nano-materials).
\end{abstract}

\section{Introduction}

Background. Neutron and X-ray reflectivity (NR and XRR) are scattering techniques based on interference. They were developed several decades ago, and still enjoy much popularity today, especially in the broad area of materials science: this includes academic and industrial problems in areas as varied as thin film magnetism, laundry detergents or bacterial membranes. The reason for this versatility lies in the unique ability of reflectivity experiments (NR or XRR) to determine the detailed structure of buried layered interfaces with nanometre precision.

Interfaces in general, typically introduce some kind of symmetry breaking in a system, which gives rise to a whole range of interesting phenomena: in the area of thin-film magnetism one finds interfacial effects such as exchange bias, exchange spring magnets, spin Hall effect, oxide heterostructures and topological insulators [1. Self-assembly at interfaces is present in soft-matter and biological systems, and is increasingly being exploited in technological applications, such as organic solar cells, laundry detergents, lubricants, cosmetic

Email address: Maximilian.Skoda@stfc.ac.uk (Maximilian W. A. Skoda)

Preprint submitted to Elsevier products, medical implants, drug delivery systems, food and so on. Sometimes, these interface-induced phenomena, such as symmetry breaking or (hierarchical) self-assembly, may give rise to 'emergent' properties or phenomena 2 .

Reflectivity and its limitations. Although many incremental improvements have been made over the years, fundamentally and with a few exceptions (e.g. spin-echo NR [3] or SERGIS [4, [5]), the type of information that can be gained from NR or XRR has not qualitatively changed, although improvements in neutron and X-ray sources, optics ("Selene" guide [6] or multiple angle-wavelength dispersive XRR [7]) and detectors (e.g. PILATUS []ㅜㄱ) have allowed for increases in available flux and thus in time resolution. Fundamentally, NR and XRR can determine the structure and composition of 'layered' systems on flat interfaces. This is achieved by measuring the reflected intensity as a function of momentum transfer $q$. This can be done by variation of the angle of incidence, the beam energy (wavelength), or both. Typically, the extracted information is in the form of scattering length density (SLD) (or electron density) as a function of distance from the interface. This density profile includes layer thicknesses, their refractive index (SLD) and

March 6, 2019 
interfacial roughness. In many cases, especially for more complex layer structures, these quantities are re-parametrised in terms of derived variables, which are more useful for the respective system (e.g. molecular density, volume fraction or area per molecule, solvent penetration, molecular tilt, interpenetration of moieties etc.).

There are two main fundamental reasons limiting the applicability of NR and XRR: The first, which applies to all reciprocal space scattering techniques,concerns the loss of phase information. This means that real space structural information cannot be obtained directly from the data (e.g. by an inverse Fourier transform). The consequence is that models need to be calculated and then fitted to the experimental data. Although there have been attempts to devise methods for 'direct inversion' 9, 10, most of these impose some restrictions to the sample composition, such as reference layers, or renders the inversion process mathematically more complicated than fitting, such that as a consequence, the uptake of these methods into the reflectivity community has been very slow. The second limitation is the requirement of (atomically) flat and smooth samples. The information content and ability to extract meaningful quantities from a reflectivity experiment is greatly reduced if the sample is non-uniform in the in-plane direction (this can be roughness, waviness or general inhomogeneity). This aspect is somewhat more important for NR, due to the typically larger illuminated sample areas. This second requirement for uniform and smooth samples thus limits the applicability of reflectivity techniques to more realistic or technologically relevant systems - a reflectivity sample will very often be an idealised version of the system one wishes to study.

On the other hand, for most soft-matter and biological systems the achievable NR or XRR resolution is not a problem, as most such layer systems have a thickness smaller than a few hundred nanometres, which is easily provided by most X-ray or neutron reflectometers.

NR and XRR are often viewed as complementary, because the fundamentals of the interaction are different: while X-rays interact with the electron shell of atoms and the scattering cross section is largely proportional to the atomic number $Z$, neutrons interact with the nuclei and their scattering cross sections are isotope specific. This provides both advantages and limitations for each of the techniques: neutrons, lacking charge, interact
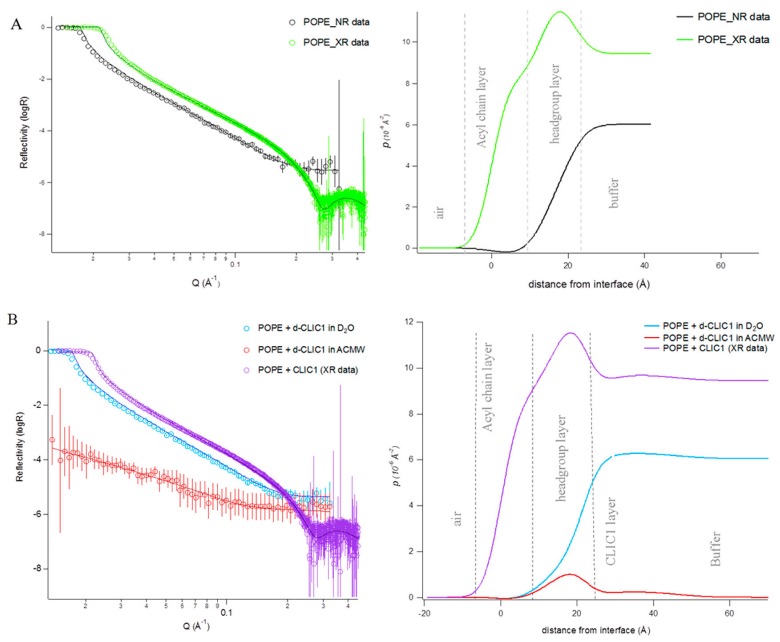

Figure 1: XRR and NR profiles, model data fits, and the scattering length density profiles of these fits described for (A) the POPE monolayer and for (B) the CLIC1 interaction with the POPE monolayer. Reprinted (adapted) with permission from 11. Copyright (2017) American Chemical Society.

only weakly with matter, which makes them very penetrating and non-destructive and well-suited for measuring buried interfaces. The isotope dependent scattering cross section makes it possible to produce scattering contrast between chemically equivalent atoms or molecules. This is most notably exploited for materials containing hydrogen, which can be substituted for deuterium. The nuclear interaction however, also gives rise to an incoherent scattering cross section, which in some cases can severely limit the dynamic range. X-ray reflectivity measurements on the other hand do not usually suffer from high incoherent backgrounds, thus providing a larger dynamic range and also - in part due to the higher flux synchrotron sources available - better signal-to-noise. But X-rays are also ionising and can lead to sample damage, while higher absorption cross sections can limit the penetration depth of the $\mathrm{X}$-ray beam, though this can usually be mitigated by using higher X-ray energies. The recent paper by Hossain et al. 11] illustrates some of the differences in dynamic range and isotopic/elemental sensitivity between XRR and NR. Fig 1] demonstrates that even a laboratory X-ray source yields an order of magnitude higher dynamic range, which allows the full first interference fringe of the monolayer sample to be visible.

Despite (or perhaps due to) the above mentioned limitations new research using XRR and NR usually 
develops along two avenues:

(a) increase in complexity

(b) increase in time resolution

The main sections of this article will be developed along these lines.

Scope and structure. Reviews on applications using neutron and X-ray reflectivity have been periodically published. Many of these focus on one technique and/or on a specific subset of scientific problems, for instance the recent reviews by Fragneto et al. 12 and Junghans et al. 13] describing advances in the study of biological membranes using neutron scattering. The article by Penfold and Thomas 14 provides a good broad overview over neutron reflectivity and small-angle scattering applied to polymer and surfactant systems, while the article by Narayanan et al. [15] reviews both neutron and X-ray scattering, but has a strong focus on bulk samples and small-angle scattering. The broad field of polymers and surfactants at interfaces using $\mathrm{NR}$ is reviewed for instance by Braun et al. 16. The benefits and limitations of using in-situ XRR for the study of protein adsorption is discussed in an article by Richter and Kuzmenko 17.

Given the breadth of applications of NR and $\mathrm{XRR}$ and the wealth of literature, it is impossible to do justice to all the published work, so the present article reviews an inevitably subjective selection of recent (approximately last 3 years) applications of $\mathrm{XRR}$ and NR in the areas soft matter and biology. In particular this article focuses on systems, which have not traditionally been studied by reflectivity measurements. It attempts to identify step changes in the capabilities of these techniques and emerging trends by considering advances in instrumentation, experimental complexity and data analysis. This is presented against the background of some inherent limitations of each technique and also in the light of the advent of new neutron and X-ray sources, such as the European Spallation Source (ESS), the SNS second target station or free electron lasers. We will endeavour to identify as yet unexplored potential of using NR and XRR for the study of soft matter and biological systems. The article is divided into two major sections: the first is reviewing recent advances in instrumentation (including sample environment and in-situ techniques). In the second part some recent research is selected to illustrate the potential future trajectory of $\mathrm{NR}$ and $\mathrm{XRR}$ in the areas of soft matter and biological systems.

\section{Technique developments}

X-ray reflectivity. While the incident flux provided by 3rd generation synchrotrons is typically one or more orders of magnitude higher than at comparable neutron sources, for the technique of reflectivity this advantage becomes somewhat diminished by the fact that most X-ray reflectometers use a monochromatic beam 1 . This means that measurements have to be performed in angle-dispersive mode, usually by changing the sample and detector angles. The requirement for movement of the sample can become the limiting factor for fast measurements. Recently, several approaches have been presented, which circumvent this problem by different means: Mocuta and co-workers 21] present an approach for fast (order of $10 \mathrm{~s}$ ) reflectivity measurements up to high momentum transfer values (close to $0.1 \mathrm{~nm}^{-1}$ ) by using an area detector an a synchronised movement of sample and detector. Using a bespoke data processing procedure, the area detector data is converted to a three-dimensional data set, from which not only the specular signal can be extracted, but also in-plane information obtained from grazing-incidence small-angle X-ray scattering (GISAXS) signal recorded. This approach uses fast-reading and low-noise area detectors and synchronous motor movements. At high-intensity Xray sources, the total acquisition time limits are fixed by the combination of the maximal motor speeds and the desired area detector acquisition speed (limiting exposure time per frame).

Lippmann and co-workers [22] use a fast rotating, slightly tilted sample reflecting to a stationary mounted position sensitive detector.The shortest measurements, covering momentum transfer values up to $0.55 \AA^{-1}$, are 2 s or less, even down to a quarter of a second. While the spatial resolution mainly depends on the beam properties and the detector pixel size, the shortest achievable acquisition time depends on the speed of the rotation table, the photon flux, and the sample detector distance.

Another method, which is similar to divergent beam methods for NR, as described in the next section, is presented by Joress et al. [23]. It is based on a polycapillary X-ray optic to produce a converging fan of radiation, the reflection of which is captured by an area detector to simultaneously collect the

\footnotetext{
${ }^{1}$ There have been successful attempts of energy-dispersive $\mathrm{XRR}$ 18, 19, 20, but this technique has not become mainstream.
} 


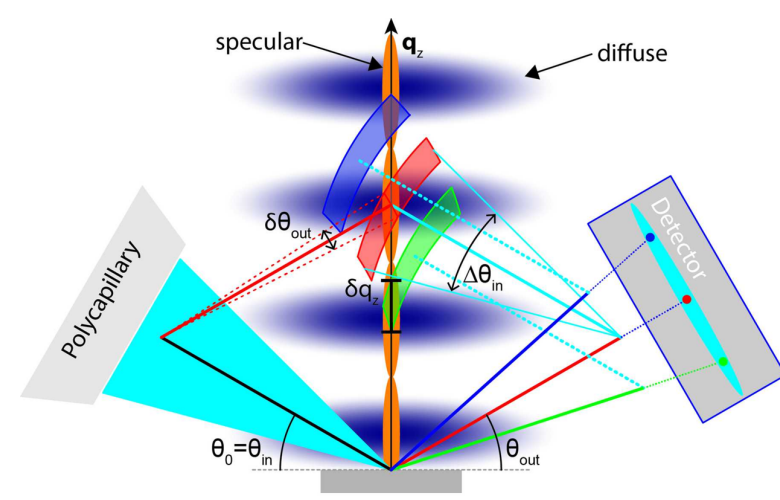

Figure 2: Schematic diagram of qXRR, from ref [23], illustrating the regions of reciprocal space probed by three particular pixels on the detector, the diffraction geometry, and its effect on the resolution. The probed regions for each of three pixels are shown in red, green and blue. Each region is bound by a set of two arcs with parallel sides corresponding to the range of incident and exit angles contributing to intensity on that pixel. The light orange features around the $\left[q_{z}\right]$ vector represent specular features. The wider, dark ellipses represent less intense diffuse features in reciprocal space. Reproduced with permission of the International Union of Crystallography https://journals .iucr.org/

XRR signal over an angular range, rather than a single angle at a time (Fig. 2, Acquisition times of the order of $1 \mathrm{~s}$ can be achieved over a momentum transfer range of up to $1.5 \AA^{-1}$, while reducing the observable $q$-range allows a time resolution down to $100 \mathrm{~ms}$.)

Festersen at al. 24 present another interesting approach, which not only allows for shorter acquisition times, but also overcomes the need for dedicated liquid surface diffractometers (with a downwards pointing beam). The authors are utilising the natural curvature of a liquid drop for variation of the angle of incidence without the need for an inclined incident beam. With this method it is possible to probe a single angle at a time (analogous to classical XRR), or a larger beam (of the order of micrometres) can be used, that is reflected into a wider angular range, enabling parallel measurements of a larger range of an XRR curve. According to the authors, their method will enable studies of liquid interfaces at sources lacking liquid diffractometers, especially ultra-fast XRR measurements via pump-probe techniques at free electron lasers.

Finally, a novel approach similar to that employed on the "RAINBOWS" reflectometer at the ILL (see next section) is presented by Tur'yanskii and co-workers [25]. The authors are using a diamond prism to decompose the spectrum of a polychromatic X-ray beam, which again largely removes the need for angular scanning. The proof-of-principle measurement shows a reflectivity curve in the momentum transfer range of 0.05 to $0.2 \AA^{-1}$ recorded in less than 5 s.

Neutron reflectivity. There have been many incremental improvements in almost all components of neutron reflectometers - from more efficient neutron guides, and more sophisticated chopper systems to much improved area detectors. But there is one feature which is becoming commonplace in almost all neutron reflectometers: focusing guides. The focusing can happen perpendicular to the scattering plane, in the scattering plane or both; it can range from relatively gentle focusing down to focusing for small sample areas of less than $1 \times 1$ $\mathrm{cm}^{2}$. The guide geometry can be either elliptical or parabolic, depending on the specific requirements and the position of the guide. Although the concept of neutron focusing is not new, the actual implementation was made possible by the advances in $2 \mathrm{D}$ position sensitive detectors providing sufficient resolution and high count rate capability, as well as by improved curved guide manufacturing techniques, providing high $m$-values ${ }^{2}$. Improved methods for processing data obtained from beams, which are divergent in the scattering plane have also been published, e.g. by Cubitt et al. [26. Describing the details of such guide systems goes beyond the scope of the present article and only few examples (not a comprehensive list) of recently upgraded neutron reflectometers are given: the already mentioned Selene guide system on the AMOR reflectometer at SINQ [27, 6], D17 at the Institut Laue-Langevin 28, the MARIA reflectometer at FRMII [29]. Similar guides for focusing in the non-scattering plane are currently underway at the ISIS spallation source for the INTER, PolRef, OffSpec and SURF reflectometers 3 Following the success of this approach, the first reflectometer at the ESS, Estia, will have a focusing guide from small samples, while more advanced reflectometer designs are already being proposed, such as the novel reflectometer design of HERITAGE 30.

One new development stands out, as it employs refractive optics, i.e. a prism, in order to separate

\footnotetext{
${ }^{2}$ The so-called m-value describes a supermirror's ability to reflect neutrons. $m$ is the ratio of the mirror's critical angle for total reflection to that of nickel $m=\frac{\theta_{\text {mirror }}}{\theta_{N i}}$

${ }^{3}$ R. Dalgliesh, M. Skoda private communication
} 
spatially a 'white' neutron beam by wavelength 31 . Once again a position sensitive detector is used to detect the different neutron wavelengths as a function of angle. This method has the advantage of largely removing the need for choppers on a reactor source, thus greatly enhancing the available flux on the sample and making this techniques ideal for fast kinetic studies. This 'RAINBOWS' system is implemented on the D50 reflectometer at ILL.

But there have also been interesting developments in methodology, such as the recent paper by Sakurai et al. 32. The authors present an extension to conventional NR, which enables the visualisation of inhomogeneous buried layers and interfaces in thin films in other words NR imaging. Unlike for X-rays, where the beam brilliance and size allows the $\mathrm{XY}$ rastering of a sample on a reasonable time scale, neutron beams cannot be collimated or focused down to $\mathrm{mm}$ or microns. Hence, the proposed method is akin to tomography, whereby the sample is rotated around the surface normal while measuring a full reflectivity profile at each step. By using Hadamard encoding, it is even possible to perform this measurement using a conventional ${ }^{3} \mathrm{He}$ tube. The collected data have to be decoded and reconstructed using well-known algorithms and similar to computed tomography, it is possible to obtain the specimen's two-dimensional neutron reflectivity distribution as one image. The spatial resolution is currently limited to $\sim 1 \mathrm{~mm}$, but this is due to the Hadamard mask slot size and could be further improved by using smaller slots or high-resolution area detectors. Although relatively time consuming ${ }^{4}$ this method would be easily applicable on high-flux neutron sources (e.g. ESS, J-PARC, SNS, ILL) and acquisition times will be further reduced by using position sensitive detectors, thus removing the need for the Hadamard encoding.

Sample environment and in-situ techniques. In addition to improvements in reflectometer design and performance, there have been equally important advances in the area of advance sample environment equipment and the use of on-beam complementary techniques. Progress in these areas is crucial for enabling new applications of reflectometry especially in the area of soft-matter and biol-

\footnotetext{
${ }^{4}$ It took the authors $42 \mathrm{~h}$ to obtain a $31 \times 31 \mathrm{~mm}^{2}$ map (1 mm resolution) and a $q$-range of up to $\sim 0.04 \AA^{-1}$ for each voxel.
}

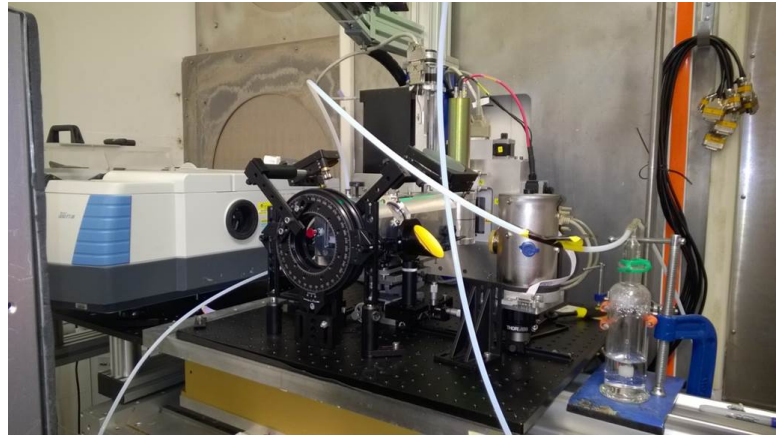

Figure 3: Combined FT-IRRAS and NR set-up on the INTER reflectometer at ISIS.

ogy: relevant, cutting edge problems in these areas often involve complex, multi-component systems with thermodynamically sensitive properties and they require the use of multiple techniques in order to obtain the full picture. A few examples are given here: Wirkert et al. 33 demonstrated the use of a high-pressure cell for in situ X-ray reflectivity measurements of liquid/solid interfaces at hydrostatic pressures up to $500 \mathrm{MPa}$ ( $5 \mathrm{kbar}$ ). This pressure regime is particularly important for the study of protein unfolding. Larsen and co-workers 34 presented a liquid sample cell for X-ray reflectivity studies with an in situ applied electrical field, which can be used to study both air-liquid and liquidliquid interfaces. It is designed to accept both copper and ITO plate electrodes, the latter being useful for simultaneous optical studies.

The BioRef II instrument 35, which has now been implemented as the new 'Spatz' instrument at ANSTO, provides a built-in attenuated total reflection (ATR) set-up, which can be used to obtain complementary, real-time molecular environment information, e.g. for studying phase transitions in lipid multilayer films. Similarly, but for the air-water interface, the recent Fourier Transform Infra-Red Reflection Absorption Spectroscopy (FT-IRRAS) add-on to the INTER reflectometer [36] at the ISIS spallation neutron source provides complementary information about surface monolayers and is able to monitor non-deuterated molecular species, which are not observable in the NR signal (Fig. 3).

Toolan and co-workers 37 have demonstrated the combination of NR with a Love wave Surface Acoustic Wave (SAW) device. By combining these techniques it is possible to determine not only the structure of the interface, but also the amount of 
void space within the thin film, which can be difficult to assess using neutron reflectivity measurements alone.

Many of the systems studied by reflectivity also lend themselves to be studied also by other methods, such as Quartz Crystal Microbalance (QCMD), ATR-FTIR or ellipsometry. These laboratory based techniques are important for several reasons: they are readily available in many research institutions and thus allow for high sample throughput, as opposed to the limited time available at large scale facilities to perform NR or XRR. In addition these techniques provide additional and sometimes complementary information about the sample (e.g. molecular environment spectroscopic information in the case of ATR-FTIR). Some measurements, e.g. QCM-D or ellipsometry can also deliver a higher time resolution than NR or XRR. Much of the precharacterisation of samples ahead of the scattering experiment is done on these bench-top instruments. However the substrates used for NR (or XRR) experiments often differ from those used in the QCM or the ATR spectrometer, which can sometimes lead to different and unexpected behaviour. Thus it makes sense to combine these techniques and be able to monitor QCM-D and/or ATR signals insitu, during the reflectivity experiment. Such arrangements are now routinely being used at different facilities. In-line QCM-D for instance is now routinely being used for solid-liquid experiments on the INTER reflectometer at ISIS.

The application of electric fields in parallel with reflectivity measurements, either for electrochemistry in solution [34, 38, or in vacuum 39, 40, has also become routine. Similarly, the study of polymer systems under confinement, by combining a hydrostatic pressure cell with NR, is still delivering useful insights (e.g. 41]), even though the technique is not new.

\section{Recent applications}

Section 2 has set the stage by describing some new developments in reflectivity instrumentation and sample environment. While advances in instrumentation mostly lead to the ability to perform measurements faster and/or over a larger $q$-range, which is useful when studying one-way kinetics, progress in sample environment including on-beam complementary techniques, is allowing researchers to tackle more complex samples.
Complexity. Neutron Reflectivity is particularly well suited for the study of layered structures at the solid-liquid interface, which makes it useful for biophysical systems. In their report from 2015 Junghans et al. 13 discuss the potential of NR for the structure determination in soft material systems, from simple supported lipid membranes in static and dynamic conditions to the adherence of complex endothelial cells under liquid shear stress. More recently, Böhm an co-workers 42] have employed a similar experimental arrangement in order to elucidate how epithelial cells adhere to a silicon substrate. Their data suggest a three-layer interfacial organization consisting of a dense protein layer with a thickness of $9 \pm 2 \mathrm{~nm}$, followed by a highly hydrated $24 \pm 4 \mathrm{~nm}$ thick layer and finally a third layer with an "average thickness of $54 \pm 18 \mathrm{~nm}$, and a huge variance, well beyond resolution effects, is associated with the composite membrane." Although the data presented is of low resolution (some measurements with $\Delta q / q=0.1$ over a limited $q$-range with a maximum of only 0.03 to $0.12 \AA^{-1}$, these experiments demonstrate the possibility of applying (neutron) reflectivity to highly complex, real systems.

In contrast to the above top down approach of studying live cells by NR, Clifton and co-workers describe the formation of realistic (asymmetric, Gram negative) bacterial membrane models [43. These models are constructed from the bottom up using Langmuir-Blodgett and Langmuir-Schaefer deposition techniques and their structure and quality is determined by NR. That these bacterial membrane models are well controlled and good mimics for real bacteria is demonstrated by the fact that they exhibit many aspects of published in-vivo behaviour. The authors present a model system in which an asymmetric bilayer floats above a phosphatidylcholine self-assembled monolayer (SAM), which itself is attached to a smooth gold surface. The asymmetric bilayer has a phospholipd (DPPC) inner and a rough lipopolysaccharide (LPS) outer leaflet. The presented system thus provides a robust platform for antibiotic development, which accessible for study not only by NR, but also by other techniques such as surface plasmon resonance, infrared spectroscopy, atomic force microscopy, and FRET.

There are many recent studies (which cannot all be cited here) which employ such models, e.g.: Michel et al. 44] study differences in the inter- 
action of active versus inactive plasticin (an antimicrobial peptide) with such membrane mimics. Dupuy et al. 45. compare two different models of the Gram negative outer membrane as well as Gram positive and eukaryotic mimics to study the mechanism of colistin (an antibiotic of last resort) interaction. The authors' findings suggest that colistin induces domains in the Gram negative inner membrane, which could lead to permeation through the domain boundaries, while for the Gram negative outer membrane, colistin remains in the headgroup region, thus displacing divalent cations, leading to outer membrane perturbation. Gram positive membrane models on the other hand are only slightly perturbed.

Also using NR, combined with ex situ ATR/FTIR, Paracini et al. 46. emphasise the importance of the physical state of the lipid matrix with regard to its interaction with antibiotics (it this case Polymyxin B). Employing a previously developed highly complex outer membrane model, the authors show that Polymyxin $\mathrm{B}$ insertion is dependent on the phase transition of LPS from the gel to the liquid crystalline state - only a tightly packed model was able to reproduce the temperature dependent disruption of the asymmetric lipid bilayer by PmB previously observed in vivo.

Other complex constructs are for instance models of the stratum corneum (SC), the outermost layer of the skin. Such model systems are of obvious interest for many reasons, such as development of transdermal drug delivery systems to personal care products. Mueller and co-workers 47] have recently developed an improved preparation method for a new stratum corneum (SC) model system, which more closely resembles natural SC compared to the more commonly used multilayer models. Their new model is particularly geared towards exploitation by $\mathrm{XRR}$ or NR: a reduction in thickness provides the opportunity to study the effects of drugs and/or hydrophilic penetration enhancers on the structure of $\mathrm{SC}$ in full detail. Indeed the same author has studied the effect of urea and taurine as hydrophilic penetration enhancers on stratum corneum lipid models [48] using - among other techniques - simultaneous infrared (attenuated total reflection, ATR) and neutron reflectivity.

Polymer thin films also lend themselves to being studied by reflectivity techniques. This research area is of course extremely broad, not least because of their use for a wide range of technological ap- plications, such as optoelectronics, drug delivery or packaging. As a consequence there is a wide variety of research being published, the exploration of which goes beyond the scope of the present review. Only the combination of reflectivity measurements of polymer films exposed to (water) vapour will be singled out, since it involves additional sample environment equipment for humidity control. Such humidity controlled sample environments are also important for research in other areas (e.g. for studying lung surfactant or ionic liquids) and as a consequence have become more widely available for use in scattering experiments. Recent examples are studies of PEDOT:PSS swelling. PEDOT:PSS is a hygroscopic conducting polymer mixture, with potential uses in solar cells, flexible optoelectronics and wearable devices. As such it is important to understand and potentially control this material's mechanical stability and electrical charge transport in presence of water vapor. Sarkar et al. [49] report the swelling kinetics and evolution of the electrical charge transport in PEDOT:PSS thin films, whose structural changes (largely thickness increase) were determined by in situ XRR. The authors observe a swelling induced microscopic rearrangement of the conducting PEDOT-rich cores in the PSS matrix, which strongly influences the electrical charge transport of the polymer film.

In contrast, Bießmann and co-workers [50] study humidity-induced changes of PEDOT:PSS electrodes by NR under high humidity conditions. The authors observe significant decrease in film swelling and water uptake when Zonyl is used as an additive (as compared to the pristine PEDOT:PSS film). This effect is further enhanced when the films are post-treated with ethylene glycol. This reduction of the water uptake is clearly beneficial when using PEDOT:PSS electrodes in organic electronic applications.

Another polymeric thin film system under humid conditions is investigated by Ederth and Ekbald [51]. The authors focus on hydrogels (highly water-soluble polymer networks), which are used in a multitude of biomedical and technological applications (e.g. where biocompatibility is required, such as implants, wound dressings or drug delivery systems, but also in bio- and other sensors). A combination of ex situ ATR-FTIR, contact angle and NR is used to characterise thickness increase and water uptake of these hydrogel films at varying relative humidities and on different substrates (gold and silica). The results show that polymer 

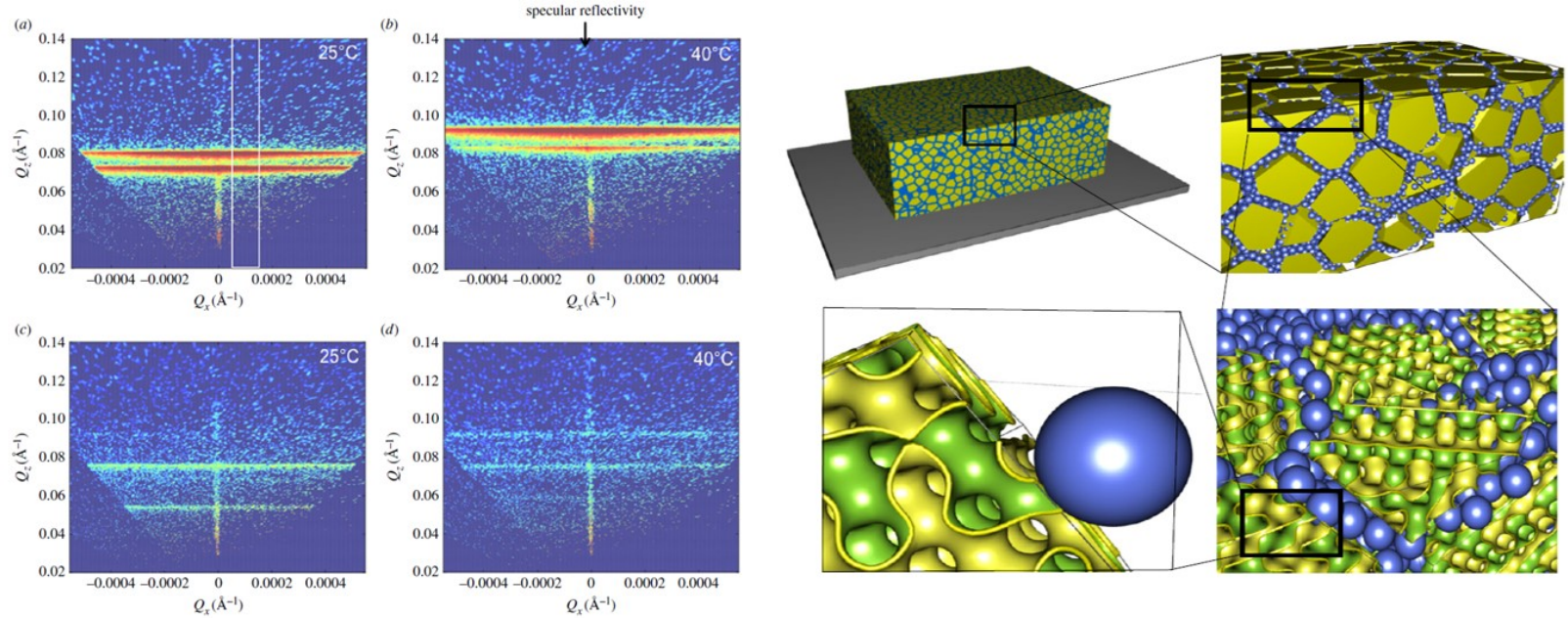

Figure 4: Off-specular NR of (a,b) lipid-only and (c,d) lipid-nanogel layers at $250^{\circ} \mathrm{C}(\mathrm{a}, \mathrm{c})$ and $40^{\circ} \mathrm{C}(\mathrm{b}, \mathrm{d})$. Inset: Proposed structural and morphological features of the mixed lipid-nanogel layer. Figure not to scale, but highlighting the main features. From ref. 52

growth proceeds at different rates and that the are structural differences on the two types of substrate.

Finally, NR (here also making use of off-specular information) is also being used to characterise even more complex architectures, where lipids under certain conditions can form not only planar bilayers, but can self-assemble into interesting three-dimensional structures. Dabkowska et al. 52 present a comprehensive study of the properties of mixed lipid layers, which are composed of glycerol monooleate and diglycerol monooleate with poly(N-isopropylacrylamide) nanogels, and can form films of reverse cubic phases that are capable of responding to temperature stimulus (Fig. 44. Such lipid-polymer (thermally) responsive layers provide the basis for the creation of new nanostructured materials with tunable properties.

Using simultaneous XRR, X-ray fluorescence near total reflection (XFNTR) and grazing incidence diffraction (GID) complemented by MD simulations, Uysal and co-workers 53. were able to elucidate details of the solvent extraction (SX) of chlorometalates from chloride solutions. The studied system is a highly concentrated solution, where ion-ion correlations and the competition between the co-ions are coupled with hydration effects and are all important in determining adsorption behaviour. For such complex systems with competing interactions only a combination of probes is able to disentangle the individual processes and provide useful insights. Another example of a related study employing a combination of NR and XRR is presented by Scoppola et al. 54. Here, ion transfer during SX is investigated by determining the structure (distributions of both extractant molecules and ions) at the liquid-liquid interface. Once again it is the combination of different contrasts in scattering and electron density which enables the determination of the potential energy across a composite liquid interface for this type of a complex system.

Using the cell developed by Wirkert et al. 33, Nowak et al. 55 have studied the structure of solidsupported lipid multilayers exposed to increased hydrostatic pressure in situ by XRR at the solid-liquid interface. The authors determined the layer vertical structure up to a maximum pressure of 4500 bar. The multilayers showed phase transitions from the fluid into different gel phases. They could also show that a pressure increase can control the transition between bi- and multilayers in situ. This switching was found to be reversible up to pressures of 750 bar, after which the initial bilayer structure could not be recovered.

Another recent example of XRR at the solidliquid interface is presented by Zhou et al. [56, 57. Using a previously developed XRR solid-liquid cell by Briscoe et al. 58 (which was initially used for the characterisation of nanofilms on mica), the authors try to further the understanding of graphene surface structure in aqueous media. They observed a thin diffuse adlayer, which they attributed to 
the formation of air bubbles on the graphene film under water. While this layer diminished after prolonged submersion in water, an additional, ca. $1 \mathrm{~nm}$ thick, layer atop graphene appeared after soaking. The authors conjecture this second layer to be due to adsorbed ion and they discuss the possible formation of a silanol layer.

Fast kinetic studies. As outlined in the introduction, the second avenue for developments in $\mathrm{X}$-ray and neutron reflectivity is that of faster measurements, in order to allow the study of a variety of time-dependent phenomena (e.g. adsorption kinetics, oxidation reactions, polymer swelling, electrochemical reactions, layer growth and many more). This section attempts to illustrate current trends and recent achievements in time-resolved reflectivity experiments by presenting a selection of recent examples.

Owing to the accessibility of the interface, timeresolved measurements at interfaces (solid or liquid) facing air or vacuum are typically easier to execute. An overview over recent advances in resolving kinetic and dynamic processes at the air-water interface using NR can be found in ref. [59]. More recently, kinetic measurements of the ageing (nitrate radical oxidation) of a monolayer model for marine aerosols (galactocerebrosides (GCB)) have been combined with in-situ IRRAS 60. (Fig. 3). The additional real-time spectroscopic information was used to determine the fate of individual molecular species in binary mixtures (palmitoleic acid (POA) and GCB), which would not be possible with NR alone. The authors used a partially deuterated GCB molecule (a sphingolipid), where only the saturated chain was deuterated. The combination of NR (sensitive only to deuterated material) and IRRAS (sensitive to both hydrogenous and deuterated species) it was possible to determine that nitrate radical oxidation was unable fully to remove a cerebroside monolayer from the surface on atmospherically relevant timescales, leaving its saturated tail at the interface. The oxidation reaction was recorded by NR with a $20 \mathrm{~s}$ time resolution over a $q$-range of 0.01 to $0.08 \AA^{-1}$.

Also at the air-water interface, Yano et al. 61. conducted time-resolved X-ray reflectivity measurements to investigate the early stage of protein adsorption and deformation at an air-water interface. The data collection time was $1.0 \mathrm{~s}$. Such investigations are important because as proteins adsorb at an air-water interface, they may lose their bio- logical activity because of subsequent denaturation, making this phenomenon important for several biological and chemical processes. The authors used three globular proteins (lysozyme, myoglobin, and bovine serum albumin (BSA)) and observed that the proteins adsorbed at the air-water interface initially possessed a thinner conformation than their native structures. The degree of unfolding for each protein was found to be inconsistent with the order of protein structural rigidity. The results however confirm earlier studies showing that protein unfolding only takes place if the kinetics of adsorption are similar to, or slower than, the kinetics of unfolding.

Reflectivity at the solid-air interface (in the area of soft-matter) is often applied to polymer thin films and in particular to the study of organic solar cell materials. The need for renewable energy sources is driving research aimed at improving the power conversion efficiency of organic solar cells, usually based on bulk heterojunctions. Both the fundamental understanding of the structure-function relationship and the improvement of stability under operational conditions are being targeted. Pont and co-workers 62 have studied the combined effects of illumination and thermal annealing on the morphological stability and photodimerization in polymer/fullerene thin films using NR. They performed kinetic measurements (at a single angle of $1^{\circ}$ ), covering a momentum transfer range of 0.01 to 0.09 $\AA^{-1}$, with $30 \mathrm{~s}$ time resolution, employing an enclosed hot stage with a steady $\mathrm{N}_{2}$ gas purge, and a white LED light source for in-situ illumination. While the authors did not find significant morphological changes with this system upon annealing up to $100{ }^{\circ} \mathrm{C}$ regardless of light exposure, they did observe that prior illumination resulted in improved stability upon high temperature annealing.

Although time-resolved studies at the solid (or liquid) to air interface are generally more readily accessible, time dependent processes at the solidliquid interface are of great importance in the areas of soft-matter and biology. Recent successful attempts to increase the complexity of lipid bilayer and related systems have been described in the previous subsection. Because of the importance of such model systems and the success in their structural determination using reflectivity, there are also studies aimed at understanding mechanisms involved in the formation of these bilayers. Koutsioubas at al. 63 have followed the formation of POPC (1-palmitoyl-2-oleoyl-sn-glycero3-phosphocholine) lipid bilayers on silica by vesicle 
fusion. NR measurements in the $q$-range of 0.02 to $0.15 \AA^{-1}$ lasted $14 \mathrm{~min}$, which allowed several frames to be recorded for a process taking a total of about $4 \mathrm{~h}$. The authors found a clear indication of stacked bilayer structure during the intermediate phase of bilayer formation. The key finding is the partial coverage of the surface by stacked bilayers at the intermediate stage of the process and also the observed desorption of lipid vesicles as the bilayer reaches completion.

Using a combination of an in-situ rheometer and $\mathrm{NR}$, it is possible to obtain the structure of surfactants (e.g. 64]) or solvated polymer brushes (e.g. 65, 66]), under constant shear flow. But even beyond that, an experiment by Adlmann and co-workers 67. demonstrates the feasibility of submillisecond time resolution for the in-depth study of reversible processes - e.g. oscillatory shear. Indeed, the authors suggest that other reversible phenomena such as magnetic relaxation, the dynamics of nematic crystals, piezoelectric behaviour and mechanical hysteresis can be probed on time scales from picoseconds to seconds. In this example, the authors also use so-called event-mode data acquisition, in which experimental metadata is stored alongside each neutron count. This method removes the need for pre-defined time intervals; in addition, data corresponding to a specific external parameter, e.g. shear rate can be co-added over many experimental cycles. Event mode data acquisition is being routinely used at many facilities.

A very interesting study of ion exchange at a solid interface is presented by Lee et al. 68... The authors present in situ time-resolved X-ray reflectivity (TXR) while utilising the resonant anomalous scattering phenomenon to obtain element-specific information through the variation in XRR contrast as a function of photon energy. Such real-time observations of ion exchange at the molecular-scale elucidate exchange reaction pathways, which are important for a broad range of chemical and transport phenomena. Specifically, the authors report temporal variation in the distribution of $\mathrm{Rb}^{+}$species at the muscovite (001)-water interface during exchange with $\mathrm{Na}^{+}$. Their TXR measurements at 25 ${ }^{\circ} \mathrm{C}$ reveal that $\mathrm{Rb}^{+}$desorption occurs over several tens of seconds during which thermodynamically stable inner-sphere $\mathrm{Rb}^{+}$slowly transforms to a less stable outer-sphere $\mathrm{Rb}^{+}$.

Finally, an XRR investigation of the electric double layer structure of an ionic liquid (DEMETFSI) modulated by an applied potential was presented by Voegeli and co-workers 69. The authors combined time-resolved XRR with cyclic voltametry, measuring XRR at short intervals of 2 and $0.3 \mathrm{~s}$ respectively. By comparing the double layer structure with the accumulated charge, the authors found evidence for structural relaxation processes that occur on a slower time scale than the accumulation of the charge at the electric double layer.

Data fitting and simulations. In addition to the already described advances in instrumentation, sample environment and the complexity of the studied systems, there are also attempts being made in terms of reflectivity data analysis, which go beyond the well-established formalisms (e.g. Parratt [70] + Névot-Croce [71] for interfacial roughness), which usually require several simplifying assumptions about the structure of the sample under study, thus applying a certain bias for the extraction and interpretation of information from the experimental data.

In the past XRR and NR data analysis was performed by fitting a slab model, which represents the individual layers in the sample, to the experimental data sets. This approach is still quite popular today owing to its relative simplicity and ease of software implementation (e.g. via the optical matrix method). For more complex sample systems that consist for instance of spatially intermixing sub-molecular groups, such an approach cannot yield meaningful physical parameters and thus limits the information that can be extracted. Models of the occupied volume across the entire interface (structure-based composition-space models) on the other hand provide the possibility to build more detailed molecular models, to directly parameterise relevant structural features and also to include additional information about the chemical and physical properties of the molecular constituents into the modeling [72, 73]. At the same time, the increased availability of high power computing and molecular modeling software has enabled the combination of reflectivity data fitting with molecular dynamics (MD) simulations (c.f [72, 173, 74, 75]). This approach - which has been reviewed in recent article by Scoppola and Schneck [76] - is mutually beneficial to both simulation and experiment: MD simulations can provide additional checks and impose limits to experimental fitting parameters, thus avoiding un-physical models; on the other hand MD-derived structures can be corrected to account 
for real world effects (e.g. lower than optimal coverage and out-of-plane membrane fluctuations).

While current mathematical and computational approaches can be used to extract physically meaningful information from reflectivity data and provide accurate estimates for errors (e.g. by using Bayesian error analysis for parameter estimation and model selection), it can also be useful to employ the same techniques in order to inform sample design and optimal measurement conditions. Very recently Treece et al. [77].. have developed a framework based on Bayesian statistics and information theory aimed at optimising the design of reflectometry experiments, in particular NR, where contrast variation plays a great role. The authors' computational model (which uses a Monte Carlo Markov chain (MCMC) simulation) is able to make predictions regarding the optimum solvent contrasts necessary, the level of statistical errors (governed by measurement time) required in a given $q$-range or the impact of spin-polarised (magnetic contrast) on the information content of a given (simulated) data set.

Other traditional approaches, e.g. with regard to roughness, are also being questioned: For example, Fujii and co-workers [78] suggest that spurious results sometimes obtained in traditional XRR calculations (especially for multilayer samples) may actually originate from the fact that diffuse scattering was not considered. The authors develop a new formalism for the reflectivity calculation taking into account the effect of attenuation in the refracted X-rays by diffuse scattering resulting from surface roughness. They also compare their new formalism with measurements of the surface roughness of the same sample by atomic force microscopy (AFM), high-resolution Rutherford backscattering spectroscopy (HRBS) and XRR and conclude that the effective roughness measured by XRR might depend on the angle of incidence.

\section{Concluding remarks}

A look at the recent evolution of X-ray and neutron reflectivity, with a focus on soft-matter and biological applications, reveals a strong drive towards faster and more complex measurements. These developments have been partly driven by scientific demand; they have been fostered by progress in instrumentation (most notably better detectors and beam optics) and have been supported by advances in computational methods (e.g. MD and MCMC simulations). It is interesting to note that both XRR and NR operate on broadly the same time scales (i.e. minutes down to seconds for one-way kinetics, down to milliseconds for cyclic processes) despite significant differences in flux and brilliance. There have been several recent demonstrations of methods for reducing count times for both NR and $\mathrm{XRR}$, involving focusing, angle multiplexing (divergent beam) or rotating samples, but these new capabilities have yet to be fully exploited.

It is also noticeable that more experiments are using combination of techniques, either in separate sessions or simultaneously, in order to extract structural details from highly complex sample systems. It is often this combination of probes which enables the detailed study of complex (layered) systems with reflectivity.

There have been many developments leading to an increased sophistication of sample environment equipment, which can be used on X-ray and neutron reflectometers. These improvements and innovations mirror the drive towards the application of $\mathrm{NR}$ and XRR to more complex and more technologically relevant or realistic sample systems. Examples of this are high pressure, humidity or electrochemistry set-ups.

In addition, MD simulations have recently become an almost routine tool for extracting and corroborating information from the scattering data, as well as reflectivity data providing validation for model systems studied in silico. On the other hand, simulation tools are commonly used for detailed and rigorous error analysis and also for informing sample design by providing a map towards a maximisation of information content for a given sample system. Despite the increased availability of versatile and user friendly software, data analysis and the extraction of meaningful information from reflectivity experiments remains one of the big hurdles which have to be overcome by new users of this technique.

The user base applying X-ray and neutron reflectivity as unique tools for accessing structural information in layered soft-matter and biological systems appears to be growing. What is more, the types of samples being approached are becoming increasingly complex and are often going beyond simplified model systems. This last development combined with an increased ease of use, both in terms of conducting the experiments and regarding the data analysis, is opening up the technique to new user communities. 


\section{Acknowledgments}

The author thanks Luke Clifton and Rebecca Welbourn for helpful discussions during the preparation of this review article and Hannah Skoda for proofreading.

\section{References}

[1] F. Hellman, A. Hoffmann, Y. Tserkovnyak, G. S. D. Beach, E. E. Fullerton, C. Leighton, A. H. MacDonald D. C. Ralph, D. A. Arena, H. A. Dürr, P. Fischer, J. Grollier, J. P. Heremans, T. Jungwirth, A. V. Kimel, B. Koopmans, I. N. Krivorotov, S. J. May, A. K. Petford-Long, J. M. Rondinelli, N. Samarth, I. K. Schuller, A. N. Slavin, M. D. Stiles, O. Tchernyshyov, A. Thiaville, B. L. Zink, Interface-induced phenomena in magnetism Rev. Mod. Phys. 89 (2017) 025006. doi:10.1103/RevModPhys.89.025006

URL https://link.aps.org/doi/10.1103/ RevModPhys.89.025006

[2] P. L. Luisi, The Emergence of Life. From Chemical Origins to Synthetic Biology., Cambridge University Press; 2 edition (October 20, 2016), 2016.

[3] W. G. Bouwman, J. Plomp, V. O. de Haan, W. H. Kraan, A. A. van Well, K. Habicht, T. Keller, M. T. Rekveldt, Real-space neutron scattering methods Nuclear Instruments and Methods in Physics Research Section A: Accelerators, Spectrometers, Detectors and Associated Equipment 586 (1) (2008) 9 - 14, proceedings of the European Workshop on Neutron Optics. doi:https://doi.org/10.1016/j.nima.2007.11.045

URL http://www.sciencedirect.com/science/ article/pii/S0168900207023807

[4] J. Major, H. Dosch, G. Felcher, K. Habicht, T. Keller, S. te Velthuis, A. Vorobiev, M. Wahl, Combining of neutron spin echo and reflectivity: a new technique for probing surface and interface order Physica B: Condensed Matter 336 (1) (2003) 8 - 15, proceedings of the Seventh International Conference on Surface X-ray and Neutron Scattering. doi:https: //doi.org/10.1016/S0921-4526(03)00264-3

URL http://www.sciencedirect.com/science/ article/pii/S0921452603002643

[5] R. Ashkar, P. Stonaha, A. Washington, V. R. Shah, M. R. Fitzsimmons, B. Maranville, C. F. Majkrzak, W. T. Lee, R. Pynn, Spin-echo resolved grazing incidence scattering (sergis) at pulsed and cw neutron sources Journal of Physics: Conference Series 251 (1) (2010) 012066.

URL http://stacks.iop.org/1742-6596/251/i=1/a= 012066

[6] C. Klauser, R. Bergmann, U. Filges, J. Stahn, A selene guide for amor Journal of Physics: Conference Series 1021 (1) (2018) 012024.

URL http://stacks . iop. org/1742-6596/1021/i=1/a= 012024
[7] T. Matsushita, E. Arakawa, W. Voegeli, Y. F. Yano, A simultaneous multiple angle-wavelength dispersive Xray reflectometer using a bent-twisted polychromator crystal Journal of Synchrotron Radiation 20 (1) (2013) 80-88. doi:10.1107/S0909049512043415 URL https ://doi .org/10.1107/S0909049512043415

[8] B. Henrich, A. Bergamaschi, C. Broennimann, R. Dinapoli, E. Eikenberry, I. Johnson, M. Kobas, P. Kraft, A. Mozzanica, B. Schmitt, Pilatus: A single photon counting pixel detector for x-ray applications Nuclear Instruments and Methods in Physics Research Section A: Accelerators, Spectrometers, Detectors and Associated Equipment 607 (1) (2009) 247 - 249, radiation Imaging Detectors 2008. doi:https://doi.org/10.1016/j.nima.2009.03.200 URL http://www.sciencedirect.com/science/ article/pii/S0168900209006573

[9] B. Bataillou, H. Moriceau, F. Rieutord, Direct inversion of interfacial reflectivity data using the Patterson function Journal of Applied Crystallography 36 (6) (2003) 1352-1355. doi:10.1107/S0021889803016649 URL https://doi .org/10.1107/S0021889803016649

[10] C. F. Majkrzak, N. F. Berk, U. A. Perez-Salas, Phasesensitive neutron reflectometry Langmuir 19 (19) (2003) 7796-7810. doi:10.1021/la0341254 URL https://doi.org/10.1021/la0341254

[11] K. R. Hossain, S. A. Holt, A. P. Le Brun, H. Al Khamici, S. M. Valenzuela, X-ray and neutron reflectivity study shows that clic1 undergoes cholesterol-dependent structural reorganization in lipid monolayers Langmuir 33 (43) (2017) 12497-12509, pMID: 29016141. arXiv: https://doi.org/10.1021/acs.langmuir.7b02872 doi:10.1021/acs.langmuir.7b02872. URL https://doi.org/10.1021/acs.langmuir. $7 \mathrm{~b} 02872$

[12] G. Fragneto, R. Delhom, L. Joly, E. Scoppola, Neutrons and model membranes: Moving towards complexity, Current Opinion in Colloid \& Interface Science 38 (2018) 108 - 121, biological Colloids and Interfaces. doi:https://doi.org/10.1016/j.cocis.2018.10.003 URL http://www.sciencedirect.com/science/ article/pii/S135902941830030X

[13] A. Junghans, E. B. Watkins, R. D. Barker, S. Singh, M. J. Waltman, H. L. Smith, L. Pocivavsek, J. Majewski, Analysis of biosurfaces by neutron reflectometry: From simple to complex interfaces, Biointerphases 10 (1) (2015) 019014. arXiv:https://doi.org/10. 1116/1.4914948 doi:10.1116/1.4914948 URL https://doi.org/10.1116/1.4914948

[14] J. Penfold, R. Thomas, Neutron reflectivity and small angle neutron scattering: An introduction and perspective on recent progress, CURRENT OPINION IN COLLOID and INTERFACE SCIENCE 19 (3) (2014) 198206.

[15] T. Narayanan, H. Wacklin, O. Konovalov, R. Lund, Recent applications of synchrotron radiation and neutrons in the study of soft matter Crystallog- 
raphy Reviews 23 (3) (2017) 160-226. arXiv: https://doi.org/10.1080/0889311X.2016.1277212

doi:10.1080/0889311X.2016.1277212

URL https://doi.org/10.1080/0889311X.2016. 1277212

[16] L. Braun, M. Uhlig, R. von Klitzing, R. A. Campbell, Polymers and surfactants at fluid interfaces studied with specular neutron reflectometry, Advances in Colloid and Interface Science 247 (2017) 130 148, dominique Langevin Festschrift: Four Decades Opening Gates in Colloid and Interface Science. doi:https://doi.org/10.1016/j.cis.2017.07.005. URL http://www.sciencedirect.com/science/ article/pii/S000186861730324X

[17] A. G. Richter, I. Kuzmenko, Using in situ x-ray reflectivity to study protein adsorption on hydrophilic and hydrophobic surfaces: Benefits and limitations, Langmuir 29 (17) (2013) 5167-5180, pMID: 23586436. arXiv:https://doi.org/10.1021/la3049532, doi:10. 1021/la3049532

URL https://doi.org/10.1021/la3049532

[18] S. Kowarik, A. Gerlach, W. Leitenberger, J. Hu, G. Witte, C. Wöll, U. Pietsch, F. Schreiber, Energydispersive $\mathrm{x}$-ray reflectivity and gid for real-time growth studies of pentacene thin films Thin Solid Films 515 (14) (2007) 5606-5610.

URL http://www.sciencedirect.com/science/ article/pii/S0040609006015240

[19] C. Weber, C. Frank, S. Bommel, T. Rukat, W. Leitenberger, P. Schäfer, F. Schreiber, S. Kowarik, Chainlength dependent growth dynamics of n-alkanes on silica investigated by energy-dispersive $\mathrm{x}$-ray reflectivity in situ and in real-time The Journal of Chemical Physics 136 (20) (2012) 204709. arXiv:https://doi.org/10. 1063/1.4719530 doi:10.1063/1.4719530 URL https ://doi .org/10.1063/1.4719530

[20] M. de Pauli, C. A. Pérez, M. C. Prado, D. H. C. Araújo, B. R. A. Neves, A. Malachias, Energy dispersive $\mathrm{x}$-ray reflectivity applied to the study of thermal stability of self-assembled organic multilayers: Results on phosphonic acids Synthetic Metals 161 (23) (2012) 2521-2525.

URL http://www.sciencedirect.com/science/ article/pii/S0379677911003973

[21] C. Mocuta, S. Stanescu, M. Gallard, A. Barbier, A. Dawiec, B. Kedjar, N. Leclercq, D. Thiaudiere, Fast X-ray reflectivity measurements using an X-ray pixel area detector at the DiffAbs beamline, Synchrotron SOLEIL Journal of Synchrotron Radiation 25 (1) (2018) 204-213. doi:10.1107/S1600577517015703 URL https://doi .org/10.1107/S1600577517015703

[22] M. Lippmann, A. Buffet, K. Pflaum, A. Ehnes, A. Ciobanu, O. H. Seeck, A new setup for high resolution fast $\mathrm{x}$-ray reflectivity data acquisition, Review of Scientific Instruments 87 (11) (2016) 113904. arXiv:https://aip.scitation.org/doi/pdf/ 10.1063/1.4967239 doi:10.1063/1.4967239 URL https://aip.scitation.org/doi/abs/10.1063/

\subsection{9}

[23] H. Joress, J. D. Brock, A. R. Woll, Quick X-ray reflectivity using monochromatic synchrotron radiation for time-resolved applications, Journal of Synchrotron Radiation 25 (3) (2018) 706-716. doi:10. 1107/S1600577518003004 URL https://doi.org/10.1107/S1600577518003004

[24] S. Festersen, S. B. Hrkac, C. T. Koops, B. Runge, T. Dane, B. M. Murphy, O. M. Magnussen, X-ray reflectivity from curved liquid interfaces, Journal of Synchrotron Radiation 25 (2) (2018) 432-438. doi: 10.1107/S1600577517018057

URL https://doi .org/10.1107/S1600577517018057

[25] A. G. Tur'yanskii, S. S. Gizha, O. V. Konovalov, Fast and ultrafast energy-dispersive x-ray reflectrometry based on prism optics JETP Letters 106 (12) (2017) 828-832.

URL https://doi.org/10.1134/S0021364017240122

Annotation: Using a diamond prism, the authors demonstrate for the first time the decomposition of the spectrum of a polychromatic beam for use in reflectivity experiments. This approach enables the fast acquisition of (X-ray) reflectivity data in a wide range of the scattering vector length $\mathrm{q}$ without angular scanning. This technique development makes it possible to study ultrafast processes in layered nanostructures. The method is similar to the one developed in ref 31 for neutron reflectivity.

[26] R. Cubitt, T. Saerbeck, R. A. Campbell, R. Barker, P. Gutfreund, An improved algorithm for reducing reflectometry data involving divergent beams or non-flat samples , Journal of Applied Crystallography 48 (6) (2015) 2006-2011. doi:10.1107/S1600576715019500. URL https : //doi .org/10.1107/S1600576715019500

[27] J. Stahn, A. Glavic, Focusing neutron reflectometry: Implementation and experience on the tof-reflectometer amor Nuclear Instruments and Methods in Physics Research Section A: Accelerators, Spectrometers, Detectors and Associated Equipment 821 (2016) 44-54. URL http://www.sciencedirect.com/science/ article/pii/S0168900216300250

[28] T. Saerbeck, R. Cubitt, A. Wildes, G. Manzin, K. H. Andersen, P. Gutfreund, Recent upgrades of the neutron reflectometer D17 at ILL, Journal of Applied Crystallography 51 (2) (2018) 249-256. doi:10.1107/ S160057671800239X

URL https://doi .org/10.1107/S160057671800239X

[29] S. Mattauch, A. Koutsioubas, U. Rücker, D. Korolkov, V. Fracassi, J. Daemen, R. Schmitz, K. Bussmann, F. Suxdorf, M. Wagener, P. Kämmerling, H. Kleines, L. Fleischhauer-Fuß, M. Bednareck, V. Ossoviy, A. Nebel, P. Stronciwilk, S. Staringer, M. Gödel, A. Richter, H. Kusche, T. Kohnke, A. Ioffe, E. Babcock, Z. Salhi, T. Bruckel, The high-intensity reflectometer of the Jülich Centre for Neutron Science: MARIA Journal of Applied Crystallography 51 (3) (2018) 646-654. doi:10.1107/S1600576718006994. 


\section{URL https://doi.org/10.1107/S1600576718006994}

[30] S. Mattauch, A. Ioffe, D. Lott, L. Bottyán, J. Daillant, M. Markó, A. Menelle, S. Sajti, T. Veres, Heritage: the concept of a giant flux neutron reflectometer for the exploration of 3-d structure of free-liquid and solid interfaces in thin films Nuclear Instruments and Methods in Physics Research Section A: Accelerators, Spectrometers, Detectors and Associated Equipment 841 (2017) 34-46.

URL http://www.sciencedirect.com/science/ article/pii/S0168900216309810

[31] R. Cubitt, J. Segura Ruiz, W. Jark, RAINBOWS: refractive analysis of the incoming neutron beam over the white spectrum. A new fast neutron reflectometry technique exploiting a focusing prism Journal of Applied Crystallography 51 (2) (2018) 257-263. doi: $10.1107 / \mathrm{S} 1600576718001528$

URL https://doi.org/10.1107/S1600576718001528

Annotation: The authors describe for the first time how a neutron prism can be used for faster neutron reflectometry, including the merging of different angles and subtraction of background. This technique is particularly useful for reactor sources, where the incident white neutron beam can be used, without the need for choppers or monochromators. The reflected beam is spatially separated according to neutron energy (or wavelength) and recorded simultaneously on a position sensitive detector. The method is similar to the one developed in ref 25 for X-ray reflectivity.

[32] K. Sakurai, J. Jiang, M. Mizusawa, T. Ito, K. Akutsu, N. Miyata, Neutron visualization of inhomogeneous buried interfaces in thin films, Scientific Reports 9 (1) (2019) 571. URL https://doi .org/10.1038/s41598-018-37094-5

[33] F. J. Wirkert, M. Paulus, J. Nase, J. Möller, S. Kujawski, C. Sternemann, M. Tolan, X-ray reflectivity measurements of liquid/solid interfaces under high hydrostatic pressure conditions, Journal of Synchrotron Radiation 21 (1) (2014) 76-81. doi:10.1107/ S1600577513021516.

URL https://doi .org/10.1107/S1600577513021516

[34] S. R. Larsen, M. Hansteen, B. Pacakova, K. Theodor, T. Arnold, A. R. Rennie, G. Helgesen, K. D. Knudsen, H. N. Bordallo, J. O. Fossum, L. P. Cavalcanti, Sample cell for studying liquid interfaces with an insitu electric field using x-ray reflectivity and application to clay particles at oil-oil interfaces Journal of Synchrotron Radiation 25 (3) (2018) 915-917. doi: $10.1107 / \mathrm{S} 1600577518004848$ URL https://doi .org/10.1107/S1600577518004848

[35] M. Strobl, R. Steitz, M. Kreuzer, M. Rose, H. Herrlich, F. Mezei, M. Grunze, R. Dahint, Bioref: A versatile time-of-flight reflectometer for soft matter applications at helmholtz-zentrum berlin Review of Scientific Instruments 82 (5) (2011) 055101. arXiv:https://doi. org/10.1063/1.3581210 doi:10.1063/1.3581210. URL https ://doi.org/10.1063/1.3581210
[36] M. W. A. Skoda, B. Thomas, M. Hagreen, F. Sebastiani, C. Pfrang, Simultaneous neutron reflectometry and infrared reflection absorption spectroscopy (irras) study of mixed monolayer reactions at the air-water interface RSC Adv. 7 (54) (2017) 34208-34214. URL http://dx.doi.org/10.1039/C7RA04900E

[37] D. T. W. Toolan, R. Barker, T. Gough, P. D. Topham, J. R. Howse, A. Glidle, Gravimetric and density profiling using the combination of surface acoustic waves and neutron reflectivity Journal of Colloid and Interface Science 487 (2017) 465-474.

URL http://www.sciencedirect.com/science/ article/pii/S0021979716308062

[38] M. Mizusawa, K. Sakurai, D. Yamazaki, M. Takeda, An electrochemical cell with vertical geometry for neutron reflectivity measurements Physica B: Condensed Matter 551 (2018) 270 - 273, the 11th International Conference on Neutron Scattering (ICNS 2017). doi:https://doi.org/10.1016/j.physb.2018.01.050 URL http://www.sciencedirect.com/science/ article/pii/S0921452618300802

[39] J. W. Dugger, L. Collins, R. J. L. Welbourn, M. W. A. Skoda, N. Balke, B. S. Lokitz, J. F. Browning, Ion movement in thin nafion films under an applied electric field Applied Physics Letters 113 (11) (2018) 113105. arXiv:https://doi.org/10.1063/1.5042211 doi:10.1063/1.5042211 URL https://doi.org/10.1063/1.5042211

[40] J. W. Dugger, W. Li, M. Chen, T. E. Long, R. J. L. Welbourn, M. W. A. Skoda, J. F. Browning, R. Kumar, B. S. Lokitz, Nanoscale resolution of electric-field induced motion in ionic diblock copolymer thin films ACS Appl. Mater. Interfaces 10 (38) (2018) 3267832687. doi:10.1021/acsami.8b11220

URL https://doi.org/10.1021/acsami.8b11220

[41] S. B. Abbott, W. M. de Vos, L. L. E. Mears, M. Skoda, R. Dalgliesh, S. Edmondson, R. M. Richardson, S. W. Prescott, Switching the interpenetration of confined asymmetric polymer brushes Macromolecules 49 (11) (2016) 4349-4357. doi:10.1021/acs.macromol.6b00310.

URL https://doi.org/10.1021/acs.macromol. $6 \mathrm{~b} 00310$

[42] P. Böhm, A. Koutsioubas, J.-F. Moulin, J. O. Rädler, E. Sackmann, B. Nickel, Probing the interface structure of adhering cells by contrast variation neutron reflectometry, Langmuir 35 (2) (2019) 513-521. arXiv: https://doi.org/10.1021/acs.langmuir.8b02228 doi:10.1021/acs.langmuir.8b02228 URL https://doi.org/10.1021/acs.langmuir. $8 \mathrm{~b} 02228$

[43] L. A. Clifton, S. A. Holt, A. V. Hughes, E. L. Daulton, W. Arunmanee, F. Heinrich, S. Khalid, D. Jefferies, T. R. Charlton, J. R. P. Webster, C. J. Kinane, J. H. Lakey, An accurate in vitro model of the e. coli envelope, Angew. Chem. Int. Ed. 54 (41) (2015) 1195211955. doi:10.1002/anie.201504287 URL https://doi.org/10.1002/anie.201504287 
[44] J. P. Michel, Y. X. Wang, I. Kiesel, Y. Gerelli, V. Rosilio, Disruption of asymmetric lipid bilayer models mimicking the outer membrane of gram-negative bacteria by an active plasticin Langmuir 33 (41) (2017) 11028-11039. doi:10.1021/acs.langmuir.7b02864. URL https://doi.org/10.1021/acs.langmuir. $7 \mathrm{~b} 02864$

[45] F. G. Dupuy, I. Pagano, K. Andenoro, M. F. Peralta, Y. Elhady, F. Heinrich, S. Tristram-Nagle, Selective interaction of colistin with lipid model membranes Biophysical Journal 114 (4) (2018) 919 - 928. doi:https://doi.org/10.1016/j.bpj.2017.12.027. URL http://www.sciencedirect.com/science/ article/pii/S0006349517351329

[46] N. Paracini, L. A. Clifton, M. W. A. Skoda, J. H. Lakey, Liquid crystalline bacterial outer membranes are critical for antibiotic susceptibility, Proceedings of the National Academy of Sciences 115 (32) (2018) E7587E7594. arXiv:https://www.pnas.org/content/115/ 32/E7587.full.pdf doi:10.1073/pnas.1803975115 URL https ://www. pnas . org/content/115/32/E7587

Annotation: Using a combination of neutron reflectivity and infrared spectroscopy, the authors highlight the importance of the physical phase when considering biological membrane mimics. They show that Polymyxin B insertion is dependent on the phase transition of LPS from the gel to the liquid crystalline state.

[47] J. Mueller, A. Schroeter, R. Steitz, M. Trapp, R. H. H. Neubert, Preparation of a new oligolamellar stratum corneum lipid model Langmuir 32 (18) (2016) 4673-4680, pMID: 27058649. arXiv: https://doi.org/10.1021/acs.langmuir.6b00655 doi:10.1021/acs.langmuir.6b00655.

URL https://doi.org/10.1021/acs.langmuir. $6 \mathrm{~b} 00655$

[48] J. Mueller, J. S. L. Oliveira, R. Barker, M. Trapp, A. Schroeter, G. Brezesinski, R. H. H. Neubert, The effect of urea and taurine as hydrophilic penetration enhancers on stratum corneum lipid models Biochimica et Biophysica Acta (BBA) - Biomembranes 1858 (9) (2016) 2006-2018.

URL http://www.sciencedirect.com/science/ article/pii/S0005273616301845

[49] B. Sarkar, M. Jaiswal, D. K. Satapathy, Swelling kinetics and electrical charge transport in PEDOT:PSS thin films exposed to water vapor Journal of Physics: Condensed Matter 30 (22) (2018) 225101. doi:10.1088/1361-648x/aabe51

URL https://doi.org/10.1088\%2F1361-648x\%

2Faabe51

[50] L. Bießmann, L. P. Kreuzer, T. Widmann, N. Hohn, J.-F. Moulin, P. Müller-Buschbaum, Monitoring the swelling behavior of pedot:pss electrodes under high humidity conditions ACS Applied Materials \& Interfaces 10 (11) (2018) 9865-9872, pMID: 29484879. arXiv:https://doi.org/10.1021/acsami. 8b00446 doi:10.1021/acsami.8b00446
URL https://doi.org/10.1021/acsami.8b00446

[51] T. Ederth, T. Ekblad, Swelling of thin poly(ethylene glycol)-containing hydrogel films in water vapor-a neutron reflectivity study, Langmuir 34 (19) (2018) 5517-5526, pMID: 29672068. arXiv: https://doi.org/10.1021/acs.langmuir.8b00177 doi:10.1021/acs. langmuir.8b00177. URL https://doi.org/10.1021/acs.langmuir. $8 \mathrm{~b} 00177$

[52] A. P. Dabkowska, M. Valldeperas, C. Hirst, C. Montis, G. K. Pálsson, M. Wang, S. Nöjd, L. Gentile, J. Barauskas, N.-J. Steinke, G. E. Schroeder-Turk, S. George, M. W. A. Skoda, T. Nylander, Non-lamellar lipid assembly at interfaces: controlling layer structure by responsive nanogel particles Interface focus 7 (28630677) (2017) 20160150-20160150.

URL https://www.ncbi.nlm.nih.gov/pmc/ PMC5474039/

[53] A. Uysal, W. Rock, B. Qiao, W. Bu, B. Lin, Twostep adsorption of ptcl62- complexes at a charged langmuir monolayer: Role of hydration and ion correlations. The Journal of Physical Chemistry C 121 (45) (2017) 25377-25383. arXiv:https://doi.org/10.1021/acs. jpcc.7b09350 doi:10.1021/acs.jpcc.7b09350 URL https://doi .org/10.1021/acs .jpcc.7b09350

Annotation: The authors employ X-ray reflectivity and in situ fluorescence measurements, thus achieving element and depth sensitivity, and they show that chlorometalate ions only adsorb in the diffuse layer at lower concentrations, while they adsorb predominantly in the Stern layer at higher concentrations.

[54] E. Scoppola, E. B. Watkins, R. A. Campbell, O. Konovalov, L. Girard, J.-F. Dufrêche, G. Ferru, G. Fragneto, O. Diat, Solvent extraction: Structure of the liquidliquid interface containing a diamide ligand Angew. Chem. Int. Ed. 55 (32) (2016) 9326-9330. doi:10.1002/ anie.201603395

URL https://doi.org/10.1002/anie.201603395

[55] B. Nowak, M. Paulus, J. Nase, P. Salmen, P. Degen, F. J. Wirkert, V. Honkimäki, M. Tolan, Solid-supported lipid multilayers under high hydrostatic pressure, Langmuir 32 (11) (2016) 2638-2643. doi:10.1021/acs. langmuir.5b04554

URL https://doi.org/10.1021/acs.langmuir. 5 b04554

[56] L. Zhou, L. Fox, M. Włodek, L. Islas, A. Slastanova, E. Robles, O. Bikondoa, R. Harniman, N. Fox, M. Cattelan, W. H. Briscoe, Surface structure of few layer graphene, Carbon 136 (2018) 255-261.

URL http://www.sciencedirect.com/science/ article/pii/S0008622318304512

[57] L. Zhou, L. Islas, N. Taylor, O. Bikondoa, E. Robles, W. H. Briscoe, Graphene surface structure in aqueous media: Evidence for an air-bubble layer and ion adsorption Carbon 143 (2019) $97-105$. doi:https: //doi.org/10.1016/j.carbon.2018.10.093 URL http://www.sciencedirect.com/science/ 
[58] W. H. Briscoe, M. Chen, I. E. Dunlop, J. Klein, J. Penfold, R. M. Jacobs, Applying grazing incidence x-ray reflectometry (xrr) to characterising nanofilms on mica Journal of Colloid and Interface Science 306 (2) (2007) 459 - 463, ghdhdfh. doi:https://doi.org/10.1016/j.jcis.2006.10.031 URL http://www.sciencedirect.com/science/ article/pii/S0021979706009611

[59] R. A. Campbell, Recent advances in resolving kinetic and dynamic processes at the air/water interface using specular neutron reflectometry, Current Opinion in Colloid \& Interface Science 37 (2018) 49 60, sI: 37C Surface Analysis Techniques 2018. doi:https://doi.org/10.1016/j.cocis.2018.06.002 URL http://www.sciencedirect.com/science/ article/pii/S1359029418300323

[60] B. Woden, M. W. A. Skoda, M. Hagreen, C. Pfrang, Night-time oxidation of a monolayer model for the air-water interface of marine aerosols - a study by simultaneous neutron reflectometry and in situ infra-red reflection absorption spectroscopy (irras), Atmosphere 9 (12). doi:10.3390/atmos9120471 URL http://www.mdpi . com/2073-4433/9/12/471

AnNotation: Using a combination of neutron reflectivity and in-situ infrared spectroscopy, the authors investigate the oxidation kinetics of binary mixtures of marine aerosol models. The additional in-situ spectroscopic information is crucial for determining residual molecular fragments left at the water interface after oxidation.

[61] Y. F. Yano, E. Arakawa, W. Voegeli, C. Kamezawa, T. Matsushita, Initial conformation of adsorbed proteins at an air-water interface J. Phys. Chem. B 122 (17) (2018) 4662-4666. doi:10.1021/acs.jpcb. $8 \mathrm{~b} 01039$

URL https://doi.org/10.1021/acs .jpcb.8b01039

Annotation: Using time-resolved X-ray reflectivity with $1 \mathrm{~s}$ time resolution, the authors study the early stages of protein adsorption and unfolding at the air-water interface. Structural changes in the initial stages of protein adsorption are interesting both from a fundamental point of view and for a wide range of bio-technical applications.

[62] S. Pont, F. Foglia, A. M. Higgins, J. R. Durrant, J. T. Cabral, Stability of polymer:pcbm thin films under competitive illumination and thermal stress Adv. Funct. Mater. 28 (40) (2018) 1802520. doi:10.1002/ adfm.201802520

URL https://doi.org/10.1002/adfm.201802520

[63] A. Koutsioubas, M.-S. Appavou, D. Lairez, Timeresolved neutron reflectivity during supported membrane formation by vesicle fusion Langmuir 33 (40) (2017) 10598-10605, pMID: 28872877. arXiv: https://doi.org/10.1021/acs.langmuir.7b02459 doi:10.1021/acs.langmuir.7b02459

URL https://doi.org/10.1021/acs.langmuir.
[64] R. J. L. Welbourn, F. Bartholomew, P. Gutfreund, S. M. Clarke, Neutron reflectometry of an anionic surfactant at the solid-liquid interface under shear, Langmuir 33 (24) (2017) 5982-5990. doi:10.1021/acs.langmuir.7b00530. URL https://doi.org/10.1021/acs.langmuir. 7b00530

[65] A. Korolkovas, P. Gutfreund, A. Chennevière, J. F. Ankner, F. A. Adlmann, M. Wolff, J.-L. Barrat, Shear deformation of low-density polymer brushes in a good solvent, Phys. Rev. E 98 (2018) 032501. doi:10.1103/ PhysRevE.98.032501

URL https://link.aps.org/doi/10.1103/PhysRevE. 98.032501

[66] A. Chennevière, F. Cousin, F. Boué, E. Drockenmuller, K. R. Shull, L. Léger, F. Restagno, Direct molecular evidence of the origin of slip of polymer melts on grafted brushes, Macromolecules 49 (6) (2016) 23482353. doi:10.1021/acs.macromol.5b02505 URL https://doi.org/10.1021/acs.macromol. $5 \mathrm{~b} 02505$

[67] F. A. Adlmann, P. Gutfreund, J. F. Ankner, J. F. Browning, A. Parizzi, B. Vacaliuc, C. E. Halbert, J. P. Rich, A. J. C. Dennison, M. Wolff, Towards neutron scattering experiments with sub-millisecond time resolution Journal of Applied Crystallography 48 (1) (2015) 220-226. doi:10.1107/S1600576714027848 URL https://doi.org/10.1107/S1600576714027848

Annotation: The authors demonstrate the feasibility of sub-millisecond time-resolved (neutron) reflectivity measurements by employing an in-situ rheometry set-up and applying oscillatory shear. This work demonstrates that by careful synchronisation of a cyclic signal, it is possible to obtain structural information by reflectivity measurements, when studying a reversible process.

[68] S. S. Lee, P. Fenter, K. L. Nagy, N. C. Sturchio, Realtime observation of cation exchange kinetics and dynamics at the muscovite-water interface Nature Communications 8 (2017) 15826.

URL https://doi.org/10.1038/ncomms15826

Annotation: Using resonant X-ray reflectivity, the authors follow the exchange of $\mathrm{Rb}+$ with $\mathrm{Na}+$ ions at the muscovite-water interface. This study combines time-resolved XRR with anomalous scattering in order to elucidate exchange reaction pathways.

[69] W. Voegeli, E. Arakawa, T. Matsushita, O. Sakata, Y. Wakabayashi, Dynamical response of the electric double layer structure of the deme-tfsi ionic liquid to potential changes observed by time-resolved x-ray reflectivity, Zeitschrift für Physikalische Chemie 230 (4) (2015) 577.

URL https://www.degruyter.com/view/j/zpch. 2016. 230.issue-4/zpch-2015-0669/zpch-2015-0669.xml

[70] L. G. Parratt, Surface studies of solids by total 
reflection of x-rays Phys. Rev. 95 (1954) 359-369 doi:10.1103/PhysRev.95.359.

URL https://link.aps.org/doi/10.1103/PhysRev. 95.359

[71] L. Névot, P. Croce, Caractérisation des surfaces par réflexion rasante de rayons $\mathrm{x}$. application à l'étude du polissage de quelques verres silicates, Rev. Phys. Appl. (Paris) 15 (3) (1980) 761-779. doi:10.1051/rphysap:01980001503076100

URL https://doi.org/10.1051/rphysap: 01980001503076100

[72] F. Heinrich, M. Lösche, Zooming in on disordered systems: Neutron reflection studies of proteins associated with fluid membranes, Biochimica et Biophysica Acta (BBA) - Biomembranes 1838 (9) (2014) 2341 - 2349, interfacially active peptides and proteins. doi:https: //doi.org/10.1016/j.bbamem.2014.03.007

URL http://www.sciencedirect.com/science/ article/pii/S0005273614001011

[73] A. V. Hughes, F. Ciesielski, A. C. Kalli, L. A. Clifton, T. R. Charlton, M. S. P. Sansom, J. R. P. Webster, On the interpretation of reflectivity data from lipid bilayers in terms of molecular-dynamics models Acta Crystallographica Section D 72 (12) (2016) 1227-1240. doi:10.1107/S2059798316016235. URL https ://doi.org/10.1107/S2059798316016235

[74] L. Darré, J. Iglesias-Fernandez, A. Kohlmeyer, H. Wacklin, C. Domene, Molecular dynamics simulations and neutron reflectivity as an effective approach to characterize biological membranes and related macromolecular assemblies Journal of Chemical Theory and Computation 11 (10) (2015) 4875-4884, pMID: 26574275. arXiv:https://doi.org/10.1021/ acs.jctc.5b00635, doi:10.1021/acs.jctc.5b00635 URL https://doi.org/10.1021/acs.jctc.5b00635

[75] A. Koutsioubas, Combined coarse-grained molecular dynamics and neutron reflectivity characterization of supported lipid membranes The Journal of Physical Chemistry B 120 (44) (2016) 11474-11483, pMID: 27748120. arXiv:https://doi.org/10.1021/ acs.jpcb.6b05433, doi:10.1021/acs.jpcb.6b05433 URL https : //doi.org/10.1021/acs.jpcb.6b05433

[76] E. Scoppola, E. Schneck, Combining scattering and computer simulation for the study of biomolecular soft interfaces Current Opinion in Colloid \& Interface Science 37 (2018) 88 100, sI: 37C Surface Analysis Techniques 2018. doi:https://doi.org/10.1016/j.cocis.2018.06.008 URL http://www.sciencedirect.com/science/ article/pii/S1359029418300049

[77] B. W. Treece, P. A. Kienzle, D. P. Hoogerheide, C. F. Majkrzak, M. Lösche, F. Heinrich, Optimization of reflectometry experiments using information theory, Journal of Applied Crystallography 52 (1) (2019) 4759. doi:10.1107/S1600576718017016 URL https://doi .org/10.1107/S1600576718017016

Annotation: The authors present a framework based on Bayesian statistics and information theory aimed at optimising the design of (neutron) reflectivity experiments. They determine the information gain from reflectivity experiments and compare these findings with established best practices and also find some non-intuitive insights. Such an approach is very useful when considering the rising complexity of sample systems, which are being investigated.

[78] Y. Fujii, Recent developments in the x-ray reflectivity analysis, American Journal of Physics and Applications 4 (2) (2016) 27-49. 